\title{
A phantom context effect: Visual phantoms enhance target visibility
}

\author{
JAMES M. BROWN and NAOMI WEISSTEIN \\ State University of New York, Buffalo, New York
}

\begin{abstract}
Moving visual phantoms may be considered an illusion in which a physically homogeneous region is perceptually segregated into figure (phantom) and ground (nonphantom) regions (Brown, 1985). The visibility of briefly flashed line segments within these identical physical regions was found to be influenced by whether or not the phantom illusion was visible. When no phantoms were seen, no difference in target visibility was evident. When phantoms were visible, target visibility was superior in phantom versus nonphantom regions of three different phantom displays. The processing of figure and ground has been hypothesized to involve specialized mechanisms in the visual pathways (Weisstein \& Wong, 1986). The results suggest that the difference in visual processing sensitivity within physically defined figure and ground regions can also occur when the perceived organization is illusory.
\end{abstract}

Moving visual phantoms (Tynan \& Sekuler, 1975) involve the perception of contours and surfaces in a homogeneous region. A common phantom-inducing stimulus is a black-and-white square-wave grating moving horizontally above and below a black homogeneous horizontal strip (see Figure 1a).

When phantoms are not visible, the homogeneous region is perceived correctly as a uniform, opaque surface occluding the inducing grating. When phantoms are seen, the apparent depth of the occluder changes, with the black grating stripes appearing complete in front of it. The organization of a phantom display is therefore ambiguous, alternating between different three-dimensional interpretations when phantoms appear and disappear. The interaction of many perceptual variables (i.e., brightness, depth, and figure-ground perception) makes phantoms a useful tool for the investigation of visual processing (see Maguire \& Brown, 1987, for a review). Some recent models (Grossberg, 1987; Grossberg \& Mingolla, 1985) have addressed the specific types of mechanisms and processes that might be involved in the alternate groupings perceived with such stimuli. The following experiments explored what happens once a phantom interpretation has been made.

With a phantom stimulus, the nonphantom portions of the occluder are seen as background to the phantom figures in front. The often striking phenomenal appearance of the phantoms as real surfaces suggests the involvement of processes normally operating in the perception of figure and ground. Recent studies by Wong and Weiss-

\footnotetext{
This research was completed by the first author as part of a dissertation in fulfiliment of requirements for a Doctor of Philosophy degree, and supported in part by Grant 84-0115 from the Air Force Office of Scientific Research to the second author. Reprint requests should be directed to the first author, Louisiana State University Medical Center, Department of Otorhinolaryngology and Biocommunication, Kresge Hearing Research Laboratory of the South, 2020 Gravier Street, Suite "A", New Orleans, LA 70112-2234.
}

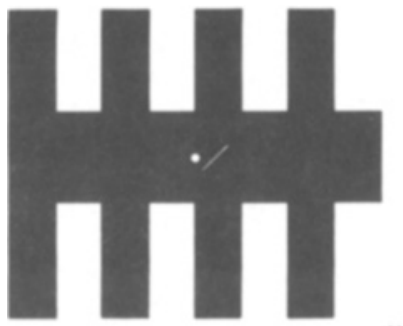

a. Block/White
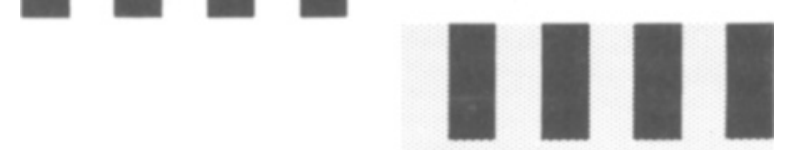

b. Gray/Black
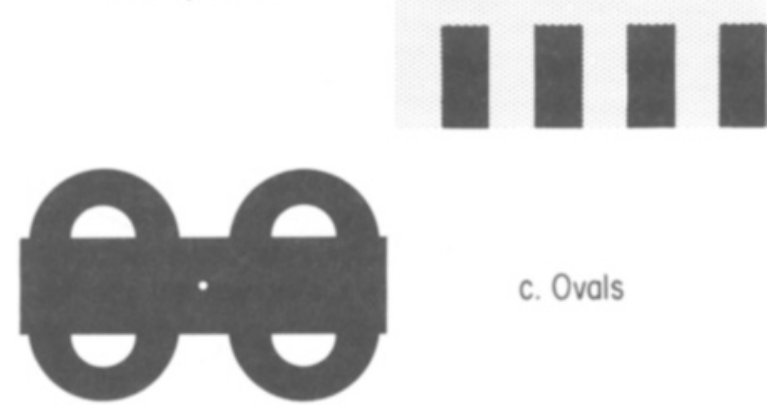

c. Ovals

Figure 1. Phantom-inducing stimuli: (a) black/white, Experiments 1 and 4; (b) gray/black, Experiment 2; and (c) ovals, Experiment 3.

tein $(1982,1983)$ have found that orientationdiscrimination performance within perceived figure regions is superior to that in perceived ground regions. Using Rubin's (1921/1958) faces/vase picture as a figureground context, they found that targets were discriminated better in the middle of the picture when it was perceived as a vase than when it was perceived as ground to the faces. This figure-ground superiority effect is thought to support a processing dichotomy within the visual system, with figure and ground analysis involving systems that 
have specific information processing characteristics (Broadbent, 1977; Julesz, 1978; Wong \& Weisstein, $1982,1983)$. In view of this processing dichotomy, we reasoned that if phantom perception and figure-ground perception were related, then using the phantom and nonphantom parts of an occluder as a figure-ground context should also produce a figure-ground superiority effect. The following experiments tested this hypothesis.

\section{EXPERIMENT 1}

\section{Method}

Subjects. Three paid naive observers and 1 of the authors (J.M.B.) participated in Experiment 1.

Apparatus. A computer (LSI-11/23) graphics system (Grinnell Image Processor) created and presented all stimuli on a black-andwhite television monitor (Setchell Carlson, 17M922). The television screen subtended $5.16^{\circ}$ visual angle (VA) high $\times 5.52^{\circ} \mathrm{VA}$ wide at a viewing distance of $2.56 \mathrm{~m}$. Inducing stimuli subtended $1.72^{\circ} \mathrm{VA}$ above and below an equally sized occluder and drifted from left to right at $0.52^{\circ} \mathrm{VA}$. Viewing, in a darkened room, was monocular (right eye preferred by all subjects) from a chin-rest. Targets were luminous line segments $0.0145^{\circ} \mathrm{VA}$ wide, $0.145^{\circ}$ VA long, tilted $45^{\circ}$ either left or right of vertical, and positioned in the middle of the occluder. An example of a right-tilted target in a phantom region is illustrated in Figure 1a.

Procedure. After dark-adapting for $5 \mathrm{~min}$, the observer adjusted television brightness and contrast for optimal phantom visibility. The general procedure consisted of their first adjusting target luminance to obtain a discrimination threshold, and then using only trials where phantoms were visible. Once threshold was obtained, a block of 80 trials was completed. Consecutive blocks of 80 trials were run until 300 trials with visible phantoms were collected.

The subjects fixated a dim fixation point at center screen. On each trial, a drifting pattern was presented for $8.6 \mathrm{sec}$. A tone cued the subjects $2.15 \mathrm{sec}$ before a target was flashed $(125 \mathrm{msec})$. Target location (either left or right of fixation, and either in a phantom or nonphantom region) and orientation (left or right) were randomized. The subjects made two responses on each trial, using the computer terminal keyboard. First, they made a two-alternative forced-choice orientation discrimination, and then a two-alternative forced-choice decision as to whether or not phantoms were visible from the time of the tone until target presentation. The subjects were instructed to guess if they were unsure of the target orientation, but not to guess whether or not they saw phantoms. In each experiment, discrimination accuracy was compared for targets flashed in phantom versus nonphantom portions of a physically homogeneous occluder.

Stimulus pattern. A black-and-white 0.9-cycles/deg square-wave grating was used to induce phantoms across a black occluder (Figure 1a). The black grating stripes appeared to complete in front of the occluder when phantoms were perceived. The white stripes and the nonphantom portions of the occluder were perceived as background to the phantoms.

\section{Results and Discussion}

The results were analyzed according to signal detection theory (Green \& Swets, 1966). For example, a left response to a target tilted left was scored as a hit, and a left response to a target tilted right was scored as a false alarm. The signal-to-noise ratio $\left(d^{\prime}\right)$ was calculated separately for each subject, for targets presented in phantom (figure) and nonphantom (ground) regions. These $d^{\prime}$ values were used as the data points in a one-way repeated

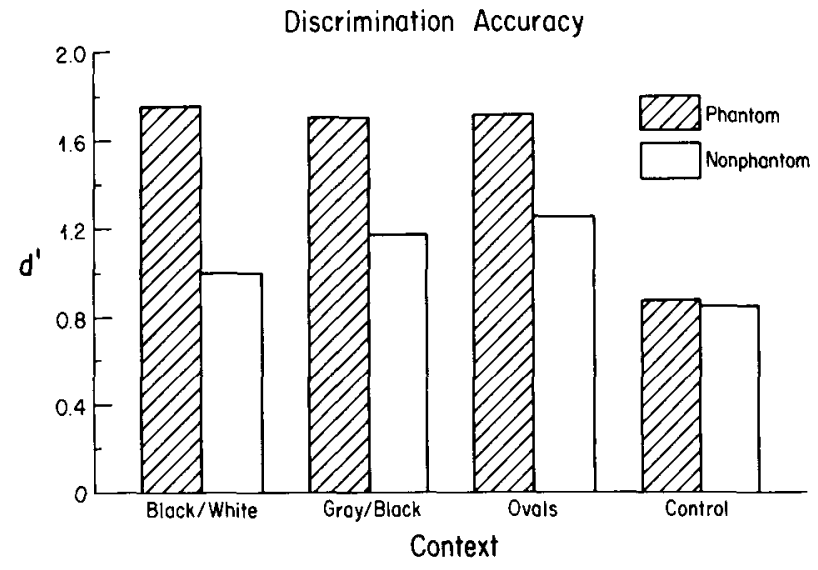

Figure 2. Discrimination accuracy for targets presented in phantom versus nonphantom regions for Experiments 1-4. Control condition evaluated performance when phantoms were not visible.

measures analysis of variance (ANOVA), which revealed a significant effect of phantom context on discrimination accuracy $[F(1,3)=10.65, p<.05]$. The signal-to-noise ratio was significantly greater for targets presented in phantom regions than those presented in nonphantom regions. The mean $d^{\prime}$ was $1.76 \pm .38$ for phantom regions and $1.00 \pm .17$ for nonphantom regions (see Figure 2).

These results suggest that the difference in discrimination accuracy between phantom and nonphantom regions is a result of their representation as figure and ground. In other words, the superior discriminability of targets in perceived phantom than in perceived nonphantom regions is analogous to findings using physically defined figure and ground regions (Wong \& Weisstein, 1982, 1983). However, before such a conclusion can be made, another possible explanation must be considered. This alternative explanation attributes the results of Experiment 1 to perceived lightness differences between the phantom and nonphantom regions.

When phantoms appear, a brightness-assimilation effect occurs between the occluder and inducing regions. In the above experiment, for example, the phantom regions appeared slightly darker than the nonphantom regions. This brightness-assimilation effect may have lead to a brightness-contrast effect between the perceived lightness of the occluder and the dimly lit targets, resulting in the targets' appearing brighter (i.e., more visible) in the phantom regions than in the nonphantom regions. The second experiment was designed to address this alternative explanation.

\section{EXPERIMENT 2}

The purpose of this experiment was to test the validity of a brightness-contrast explanation for the superior discriminability of the targets in the preceding experiment. A new stimulus of opposite contrast was created such that when phantoms were visible, the nonphantom parts of the occluder now appeared slightly darker than the phantom 
parts. Thus, a brightness-contrast explanation would predict results opposite those of a figure-ground explanation. The only difference in the method was the addition of 2 more observers.

\section{Method}

In addition to the 4 observers used in Experiment 1, 2 more paid naive observers were used in Experiment 2 . The apparatus and procedure were the same as in Experiment 1.

The stimulus pattern used to induce phantoms across a gray occluder (see Figure 1b) was a gray-and-black square-wave grating. The spatial and temporal parameters of the inducing pattern were the same as in Experiment 1. The gray stripes appeared complete in front of the occluder when phantoms were perceived, with the black stripes and the nonphantom portions of the occluder perceived as background to the phantoms. The nonphantom portions of the occluder also appeared darker than the phantom portions.

\section{Results and Discussion}

Again, $d^{\prime}$ was calculated separately for each subject for targets presented in phantom and nonphantom regions. These $d^{\prime}$ values were used in a repeated measures ANOVA that revealed a significant effect of phantom context $[F(1,5)=28.33, p<.01]$. The mean $d^{\prime}$ was $1.70 \pm .19$ in phantom regions and $1.17 \pm .24$ in nonphantom regions (see Figure 2). These results safely rule out a brightness-contrast explanation while also reinforcing a figure-ground theory. The next experiment tested the generalizability of the phantom context effect by using an inducing stimulus that was not a grating.

\section{EXPERIMENT 3}

The enhanced discriminability of targets in phantom versus nonphantom parts of the occluders in Experiments 1 and 2 was found using the same spatial-frequency square-wave inducing grating. Despite the different lightness relationships of those gratings, the particular form of the inducing stimuli was the same and, although unlikely, may have been contributing to the effect. This experiment used a completely new inducing stimulus that also produced vivid phantoms.

\section{Method}

The original 4 observers used in Experiment 1 were used in Experiment 3. The apparatus and procedure were the same as in Experiment 1 .

The stimulus pattern used in this experiment was an ovals inducing pattern (Figure 1c). The width of the arches was the same as the width of the black grating bars in the two previous experiments. When phantoms are seen with this pattern, the incomplete arches are perceived as complete ovals in front of the occluder.

\section{Results and Discussion}

The influence of phantom context was again significant as revealed by a repeated measures ANOVA comparing the $d^{\prime}$ s from phantom and nonphantom regions $[F(1,3)$ $=11.03, p<.05]$. The mean $d^{\prime}$ for targets in phantom regions was $1.72 \pm .10$; in nonphantom regions it was $1.25 \pm .22$ (see Figure 2). These results indicate that the consistent effect of a phantom context on discrimination accuracy found in all three experiments was probably due to the perception of phantoms and their organization as figure regions, and not the inducing pattern per se.

\section{EXPERIMENT 4}

The apparently consistent effect of phantom perception on target visibility suggests a perceptual influence on what might be considered an earlier process orientation discrimination. However, until performance is assessed while the phantom illusion is absent, this conclusion may be premature. As a control condition, this experiment compared target visibility within the physically identical phantom and nonphantom regions when the illusion was not visible. A moving pattern was so effective at inducing the illusion that a stationary pattern was used in this experiment to reduce the number of trials on which phantoms were seen. Phantoms are visible with a stationary inducing pattern, but typically less often and less saliently (Gyoba, 1983).

\section{Method}

Five subjects participated in the experiment, 3 new ones and 2 from the previous experiments.

The pattern was the same as that used in Experiment 1 (Figure 1a). The apparatus and viewing conditions were also the same. To present the "phantom" regions both left and right of fixation and to avoid any movement, real or apparent, while changing their position, the stimulus pattern was blanked to gray between trials. The occluder and fixation point were always present. A trial started with the pattern appearing on the screen. After $2 \mathrm{sec}$, a tone warned the subjects that a target would appear in $2 \mathrm{sec}$. Target location and orientation were again randomized. The pattern was removed after the subject responded. The subjects' responses were the same as before. First, discrimination threshold was set, using only trials when phantoms were not visible. After threshold was obtained, a total of 300 trials when phantoms were not visible were collected.

\section{Results and Discussion}

A repeated measures ANOVA revealed no difference in $d^{\prime}$ for targets presented in phantom versus nonphantom parts of the occluder. The mean $d^{\prime}$ for targets was $.87 \pm .26$ in phantom regions and $.84 \pm .35$ in nonphantom regions (see Figure 2). These results are opposite those of Experiment 1 when phantoms were visible with the same stimulus pattern. It appears safe to conclude that the difference in target visibility found in Experiments 1-3 was due to the perception of phantoms.

\section{GENERAL DISCUSSION}

The purpose of the present experiments was to test for a figure-ground superiority effect (Wong $\&$ Weisstein, 1982) using the phantom and nonphantom portions of occluders as figure-ground contexts. The results showed that orientation-discrimination performance was superior for targets presented in phantom, as opposed to nonphantom, portions of physically homogeneous occluders. This phantom-superiority effect is unlike such other contextsuperiority effects as the word-superiority effect (Baron, 
1981), the object-superiority effect (Weisstein \& Harris, 1974), and the figure-ground superiority effect of Wong and Weisstein (1982) because no physical context was present. The phenomenal appearance of phantoms alternating with a background on a physically homogeneous occluder was the only difference between the regions. Therefore, the difference in performance in each experiment cannot be due to changes or differences in the physical stimulus. Considering the perception of phantoms as a figure-ground phenomenon, however, does reveal a plausible explanation for these performance differences.

It has been proposed that figure and ground analysis is carried out by systems with different information processing characteristics (Broadbent, 1977; Julesz, 1978; Weisstein \& Wong, 1986; Wong \& Weisstein, 1982, 1983). Figure processing is assumed to involve mechanisms most sensitive to high-spatial- and low-temporalfrequency information, and ground processing is assumed to involve mechanisms most sensitive to low-spatial- and high-temporal-frequency information. Due to their spatial and temporal sensitivities, the figure system is thought to be most sensitive to detail information (Breitmeyer \& Ganz, 1976; Broadbent, 1977; Weisstein, Ozog, \& Szoc, 1975 ) whereas the ground system is thought to function as a low-resolution "early warning system" (Julesz, 1978). The superior discriminability and detectability of targets in perceived figure versus perceived ground regions has been attributed to this functional specialization (Wong \& Weisstein, 1982, 1983). The figure system is better equipped for an orientation-discrimination task, a task requiring fine detail information. This logic is extended one step further with the phantom results reported here. The phantom (i.e., perceived-figure) regions are being processed by the figure system, resulting in enhanced orientation-discrimination performance in those areas. The ground system, most sensitive to lowspatial-frequency information, is not equipped for such a high-resolution task, resulting in the poor discrimination performance in the nonphantom (i.e., perceivedground) regions. When phantoms are visible, it appears that the visual system not only supplies the missing contour and surface information consistent with that figureground organization, but also processes these illusory figure and ground regions as though physically defined figure and ground regions were present.

In conclusion, by using the perceived phantom and nonphantom regions of homogeneous occluders as figureground contexts, we have attempted to show a connection between phantom perception and figure-ground perception. These illusory regions appeared to function as physically defined figure-ground regions by producing an effect on orientation-discrimination performance analogous to the figure-ground superiority effect (Wong \& Weisstein, 1982, 1983). Our results, and other phantom studies showing specific interactions of depth information with phantom visibility (Brown, 1985; Weisstein,
Maguire, \& Williams, 1982), support an interpretation of phantom perception as a figure-ground phenomenon. This approach may prove useful in the search for a theoretical understanding of phantoms and in providing further information about the interaction of perceptual organization and early visual processing.

\section{REFERENCES}

BARON, J. (1981). The word superiority effect. In W. K. Estes (Ed.), Handbook of learning and cognitive processes (Vol. 6, pp. 317-364). Hillsdale, NJ: Erlbaum.

BREITMEYER, B, \& GANZ, L. (1976). Implications of transient and sustained channels for theories of visual pattern masking, saccadic suppression and information processing. Psychological Review, 83, 1-36.

Broadbent, D. E. (1977). The hidden preattentive process. American Psychologist, 32, 109-118.

Brown, J. M. (1985). Phantom contour completion: A figure/ground approach. Unpublished doctoral dissertation, State University of New York at Buffalo.

Green, D. M., \& Swets, J. A. (1966). Signal detection theory and psychophysics. New York: Wiley.

Grossberg, S. (1987). Cortical dynamics of three-dimensional form, color, and brightness perception: 1. Monocular theory. Perception \& Psychophysics, 41, 87-116.

GrossberG, S., \& Mingolla, E. (1985). Neural dynamics of form perception: Boundary completion, illusory figures, and neon color spreading. Psychological Review, 92, 173-211.

GrobA, J. (1983). Stationary phantoms: A complete effect without motion and flicker. Vision Research, 23, 205-211

Julesz, B. (1978). Perceptual limits of texture discrimination and their implications to figure-ground separation. In E. Leeuwenberg \& $\mathrm{H}$. Buffart (Eds.), Formal theories of perception (pp. 205-216). New York: Wiley.

Maguire, W., \& Brown, J. M. (1987). The current state of research into visual phantoms. In S. Petry \& G. Meyer (Eds.), The perception of illusory contours (pp. 213-219). New York: Springer-Verlag

RuBin, E. (1958). Figure and ground. In D. C. Beardslee \& M. Wertheimer (Eds.), Readings in perception (pp. 194-203). New York: Van Norstrand. (Original work published 1921)

Tynan, P., \& Sekuler, R. (1975). Moving visual phantoms: A new contour completion effect. Science, 188, 951-952.

WeIsSteIN, N., \& HaRRIS, C. S. (1974). Visual detection of line segments: An object superiority effect. Science, 186, 752-755.

Weisstein, N., Maguire, W., \& Williams, M. C. (1982). The effect of perceived depth on phantoms and the phantom motion aftereffect. In J. Beck (Ed.), Organization and representation in perception (pp. 235-249). Hillsdale, NJ: Erlbaum.

Weisstein, N., OzoG, G., \& Szoc, R. A. (1975). A comparison and elaboration of two models of metacontrast. Psychological Review, 82, 325-343.

Weisstein, N., \& Wong, E. (1986). Figure-ground organization and the spatial and temporal responses of the visual sysem. In $\mathrm{H}$. Nusbaum \& E. Schwab (Eds.), Pattern recognition by humans and machines: Vol. 2. Visual perception (pp. 31-64). New York: Acadernic Press.

WONG, E., \& WeissteIN, N. (1982). A new perceptual contextsuperiority effect: Line segments are more visible against a figure than against a ground. Science, 218, 587-589.

WoNG, E., \& WeIsstein, N. (1983). Sharp targets are detected better against a figure and blurred targets are detected better against a background. Journal of Experimental Psychology: Human Perception \& Performance, 9, 194-202.

(Manuscript received September 22, 1986; revision accepted for publication June $24,1987$. ) 